\title{
On the Livingstone-Wagner Theorem
}

\author{
V. B. Mnukhin \\ Department of Mathematics and Computer Science \\ University of the South Pacific, Suva, Fiji Islands. \\ mnukhin_v@usp.ac.fj \\ I. J. Siemons \\ School of Mathematics, University of East Anglia \\ Norwich NR4 7TJ, United Kingdom. \\ j.siemons@uea.ac.uk
}

Submitted: May 16, 2002; Accepted: Mar 30, 2004; Published: Apr 6, 2004

MR Subject Classifications: 20B25, 52A25

\begin{abstract}
Let $G$ be a permutation group on the set $\Omega$ and let $\mathcal{S}$ be a collection of subsets of $\Omega$, all of size $\geq m$ for some integer $m$. For $s \leq m$ let $n_{s}(G, \mathcal{S})$ be the number of $G$-orbits on the subsets of $\Omega$ which have a representative $y \subseteq x$ with $|y|=s$ and $y \subseteq x$ for some $x \in \mathcal{S}$. We prove that if $s<t$ with $s+t \leq m$ then $n_{s}(G, \mathcal{S}) \leq n_{t}(G, \mathcal{S})$. A special case of this theorem is the Livingstone-Wagner Theorem when $\mathcal{S}=\{\Omega\}$. We show how the result can be applied to estimate orbit numbers for simplicial complexes, sequences, graphs and amalgamation classes. It is also shown how this theorem can be extended to orbit theorems on more general partially ordered sets.
\end{abstract}

\section{Orbit TheOREMS}

Let $(G, \Omega)$ be a permutation group on an arbitrary set $\Omega$ and let $k \leq|\Omega|$ be an integer. Then $G$ acts on the collection $\Omega^{\{k\}}$ of all $k$-element subsets of $\Omega$. If $y \in \Omega^{\{k\}}$ then we denote the orbit of $y$ by $y^{G}$. If $\mathcal{S}$ is a family of subsets of $\Omega$ then we put $n_{k}(G, \mathcal{S}):=\mid\left\{y^{G}: y \in \Omega^{\{k\}}\right.$ and $y \subseteq x$ for some $\left.x \in \mathcal{S}\right\} \mid$. Further, $x$ is maximal in $\mathcal{S}$ if $x \subseteq x^{\prime} \in \mathcal{S}$ implies that $x=x^{\prime}$ and we put $\mathrm{m}(\mathcal{S}):=\min \{|x|: x$ is maximal in $\mathcal{S}\}$. 
Main TheOrem Let $(G, \Omega)$ be a permutation group and let $\mathcal{S}$ be a family of subsets of $\Omega$. If $s<t$ are integers with $s+t \leq \mathrm{m}(\mathcal{S})$ then $n_{s}(G, \mathcal{S}) \leq n_{t}(G, \mathcal{S})$.

The purpose of this note is to bring together various results in combinatorics which are all linked to each other via this theorem. In the first instance we should mention the theorem of Livingstone and Wagner [13] on the orbits of permutation groups when acting on subsets: this is the particular case when $\mathcal{S}=\{\Omega\}$. There are however many other applications of the theorem in combinatorial topology, graph theory and other parts of combinatorics which are new or simplify existing proofs. These applications will be stated first. In Section 2 we prove a more general orbit theorem on automorphism groups of partially ordered sets which contains the Main Theorem as a special case. There we also provide the proofs of the corollaries. The most interesting open question is: What else can be said about the sequence $n_{s}(G, \mathcal{S}), n_{s+1}(G, \mathcal{S}), \ldots$, in particular when $s$ belongs to the upper part of the interval $1 \leq s \leq \mathrm{m}(\mathcal{S})$ ? The importance of this question can be seen from the following comments on simplicial geometry.

1. Simplicial Complexes: If $\mathcal{K}$ is a collection of finite subsets of a vertex set $\Omega$ then $\mathcal{K}$ is a simplicial complex on $\Omega$ if $x \in \mathcal{K}$ and $y \subseteq x$ implies that $y \in \mathcal{K}$. Each $x \in \mathcal{K}$ is called a face of $\mathcal{K}$ and a maximal face (with regard to inclusion) is a facet of $\mathcal{K}$. The dimension $d(x)$ of $x \in \mathcal{K}$ is $|x|-1$ and $\mathcal{K}$ is pure of dimension $d$ if all facets have dimension $d$. For arbitrary complexes we put $\delta(\mathcal{K}):=\min \{d(x): x$ is a facet of $\mathcal{K}\}$. Thus if we take for $\mathcal{S}$ the set of all facets of $\mathcal{K}$ then the Main Theorem takes the form

TheOREm 1.1 Let $\mathcal{K}$ be a simplicial complex and $G \subseteq$ Aut $\mathcal{K}$. If $s<t$ are integers with $s+t \leq \delta(\mathcal{K})-1$ then the number of $G$-orbits on s-dimensional faces is at most the number of $G$-obits on $t$-dimensional faces.

We may therefore review some of the standard results from simplicial topology. If $\mathcal{K}$ is finite then its face vector is $\left(f_{0}, f_{1}, \ldots\right)$ where $f_{k}:=|\{x \in \mathcal{K}: d(x)=k\}|$. If $G=1$ in Theorem 1.1 we see that $f_{s} \leq f_{t}$ for all $s<t$ with $s+t \leq \delta(\mathcal{K})-1$. This extends Hibi's theorem [12] to complexes which may not be pure. For the substantial research on face vectors see $[4,19,17,5,2]$ and Chapter 18 in [10]. In the late 1950's Motzkin and independently Welsh conjectured that the face vector of a convex polytope is unimodal, in the sense that $f_{0} \leq f_{1} \leq \cdots \leq f_{k} \geq f_{k+1} \geq \cdots \geq f_{d}$ for some $k$ with $0 \leq k \leq d$, see [18,3]. This was disproved by Björner [3] who found a 20 -dimensional simplicial polytope on about $4.2 \times 10^{12}$ vertices for which $f_{11}>f_{12}<f_{13}$. The condition in the Main Theorem on $s+t$ can therefore not be relaxed in general. However, there are results on the face numbers $f_{s}$ for the upper range of the interval $0 \leq s \leq \delta(\mathcal{K})$ if additional assumptions on $\mathcal{K}$ are made. For 
instance, if $\mathcal{K}$ is a pure complex in which the Dehn-Sommerville equations hold (for example if $\mathcal{K}$ is a triangulation of a sphere or a Gorenstein complex, see [5]), then Björner [3, 4] showed that

$$
f_{0}<f_{1}<\cdots<f_{\lceil d+1 / 2\rceil-1} \leq f_{\lceil d+1 / 2\rceil} \quad \text { and } \quad f_{\lceil 3 d / 4\rceil}>\cdots>f_{d-1}>f_{d}
$$

where $d=\delta(\mathcal{K})$. It would therefore be interesting to investigate to which extent such inequalities for the face vector can be extended to orbits, and furthermore, if topological conditions such as the Dehn-Sommerville equations can be extended meaningfully to permutation groups in general.

2. Sequences: Let $A:=\left\{\alpha_{0}, \alpha_{1}, \ldots\right\}$ be some non-empty alphabet and let $x=\left(x_{1}, x_{2}, \ldots, x_{n}\right)$ with $x_{i} \in A$ be a word in $A$, of length $\|x\|=n$. For any index set $J \subseteq\{1, \ldots, n\}$ we may form the subword $x^{J}$ obtained by deleting from $x$ all letters $x_{j}$ with $j \in J$. In particular, the length of $x^{J}$ is $\left\|x^{J}\right\|=\|x\|-|J|$. If $j \leq\|x\|$ let $n_{j}(x):=\mid\left\{x^{J}: J \subseteq\{1, \ldots, n\}\right.$ and $\left.\left\|x^{J}\right\|=j\right\} \mid$ be the number of distinct subwords of $x$ of length $j$.

THEOREM 1.2 Let $x$ be a word of finite length in some non-empty alphabet. If $s<t$ are integers with $s+t \leq\|x\|$ then $n_{s}(x) \leq n_{t}(x)$.

The connection to the Main Theorem comes from a group action on an infinite amalgamation class, this will be explained in Section 2. Here again, it would obviously be nice to be able to say something about $n_{s}(x), n_{s+1}(x), \ldots$ when $2 s>\|x\|$. By computer search we have examined all binary words of length $\leq 10$ : in all cases the subword distribution $n_{0}(x), n_{1}(x), \ldots$ is unimodal and somewhat similar to the distribution above derived from the Dehn-Sommerville equations. However, we have not been able to prove that these properties hold in general.

3. Graphs: Let $\Gamma=(V, E)$ be a graph with vertex set $V$ and edge set $E \subseteq$ $V^{\{2\}}$. We are interested in the subgraphs of $\Gamma$ that are obtained by deleting vertices or edges from $\Gamma$. More precisely, we say that $\Gamma^{\prime}=\left(V^{\prime}, E^{\prime}\right)$ is a vertex deleted subgraph of $\Gamma$ if $V^{\prime} \subset V$ and $E^{\prime}=E \cap V^{\prime\{2\}}$.

TheOrem 1.3 Let $\Gamma=(V, E)$ be a graph on $v \leq \infty$ vertices. For $s \leq v$ let $n_{s}(\Gamma, V)$ denote the number of isomorphism classes of vertex deleted subgraphs $\left(V^{\prime}, E^{\prime}\right)$ of $\Gamma$ with $\left|V^{\prime}\right|=s$. If $s<t$ are integers with $s+t \leq v$ then $n_{s}(\Gamma, V) \leq$ $n_{t}(\Gamma, V)$.

Similarly, we say that $\Gamma^{\prime}=\left(V^{\prime}, E^{\prime}\right)$ is an edge deleted subgraph of $\Gamma$ if $V^{\prime}=V$ and $E^{\prime} \subseteq E$. 
TheOrem 1.4 Let $\Gamma=(V, E)$ be a graph with $e \leq \infty$ edges. For $s \leq e$ let $n_{s}(\Gamma, E)$ denote the number of isomorphism classes of edge deleted subgraphs $\left(V, E^{\prime}\right)$ of $\Gamma$ with $\left|E^{\prime}\right|=s$. If $s<t$ are integers with $s+t \leq e$ then $n_{s}(\Gamma, E) \leq n_{t}(\Gamma, E)$.

Apart from this nothing appears to be known about the $n_{s}(\Gamma, E)$ when $2 s>e+1$. In fact, we conjecture that this sequence is unimodal for any finite graph, but we have not been able to prove this assertion.

Another application concerns the chromatic polynomial of a graph. Let $\Gamma$ be a finite graph and $\chi(\Gamma, x)=c_{n} x^{n}-c_{n-1} x^{n-1}+\cdots+(-1)^{n} c_{0}$ its chromatic polynomial. It is well known [6] that the coefficients $c_{i}$ are the coordinates of the face vector of the broken circuit complex. So Theorem 1.1 implies that the $c_{0}, c_{1}, \ldots, c_{n}$ are initially non-decreasing. For graphs this result was proved by different methods in 1972 by Heron [11], see also [1, Proposition 14.6]. In 1968 Read [15] conjectured that $c_{0}, c_{1}, \ldots, c_{n}$ is unimodal. This conjecture has remained open.

\section{Orbits in Partially Ordered Sets}

2.1 Two General Orbit Theorems: We set out to investigate orbits in partially ordered sets more generally. Let $(P, \leq)$ be a ranked partially ordered set. We denote the set of all elements $y$ of rank $r(y)=k$ by $P_{k}$. If $\mathcal{S} \subseteq P$ we set $P^{\mathcal{S}}:=\{y \in P: y \leq x$ for some $x \in \mathcal{S}\}$ and we write $P_{k}^{\mathcal{S}}$ instead of $P^{\mathcal{S}} \cap P_{k}=\left(P^{\mathcal{S}}\right)_{k}$. Furthermore, $P$ is locally finite if $P^{\mathcal{S}}$ is finite for all finite subsets $\mathcal{S}$ of $P$.

Let $F$ be a field of characteristic 0 and let $M=F P$ be the vector space over $F$ with basis $P$. For any integer $k \geq 0$ let $M_{k}=F P_{k}$ be the subspace with basis $P_{k}$. We set $M_{k}=0$ for $k<0$. If $\mathcal{S} \subseteq P$ let $M^{\mathcal{S}}$ and $M_{k}^{\mathcal{S}}$ denote the subspaces of $M$ with basis $P^{\mathcal{S}}$ and $P_{k}^{\mathcal{S}}$ respectively.

The group of all automorphisms of $(P, \leq)$ is denoted by Aut $(P)$. For $G \subseteq$ Aut $(P)$ and $x \in P$ the orbit $\left\{x^{g}: g \in G\right\}$ is denoted by $x^{G}$. For $\mathcal{S} \subseteq P$ and $k \in \mathbb{N}$ the number of $G$-orbits $x^{G}$ with $x \in P^{\mathcal{S}}$ is denoted by $n_{k}(G, \mathcal{S}):=\left|\left\{x^{G}: x \in P_{k}^{\mathcal{S}}\right\}\right|$. Consider the subspace $A_{k}^{G} \subseteq M_{k}$ given by

$$
A_{k}^{G}=\left\langle x-x^{g}: x \in P_{k}, g \in G\right\rangle .
$$

In this expression it is sufficient to select just one arbitrary representative in $P_{k}$ for each $G$-orbit: For if $x^{\prime}=x^{h}$ then $x^{\prime}-x^{\prime g}=-\left(x-x^{h}\right)+\left(x-x^{h g}\right)$. The following general lemma is of independent interest: 
Lemma 2.1 (i) If $G \subseteq \operatorname{Aut~}(P)$ and $k \geq 0$ then $\left|\left\{x^{G}: x \in P_{k}\right\}\right|=\operatorname{dim} M_{k} / A_{k}^{G}$. (ii) Let $\mathcal{S} \subseteq P$. Then the dimension of $\left\langle x+A_{k}^{G}: x \in P_{k}^{\mathcal{S}}\right\rangle \subseteq M_{k} / A_{k}^{G}$ is $n_{k}(G, \mathcal{S})$.

Proof: From the definition it follows easily that $x+A_{k}^{G}=x^{\prime}+A_{k}^{G}$ with $x, x^{\prime} \in$ $P_{k}$ if and only if $x$ and $x^{\prime}$ are in the same orbit. Thus $\left\{x^{G}: x \in P_{k}\right\}$ is in bijection with $\left\{x+A_{k}^{G}: x \in P_{k}\right\}$. To show that the latter set is linearly independent suppose that $x_{1}+A_{k}^{G}, x_{2}+A_{k}^{G}, \ldots$ are pairwise distinct and let $\lambda_{1}\left(x_{1}+A_{k}^{G}\right)+\lambda_{2}\left(x_{2}+A_{k}^{G}\right)+\cdots=A_{k}^{G}$. Thus $\lambda_{1} x_{1}+\lambda_{2} x_{2}+\cdots \in A_{k}^{G}$. Then, by the comment above, we write $\sum \lambda_{i} x_{i}=\sum \alpha_{i j}\left(x_{i}-x_{i j}\right)$ where for each $i$ the $x_{i j} \neq x_{i}$ are certain distinct elements in the same orbit as $x_{i}$. But then $\alpha_{i j}=0=\lambda_{i}$ for all $i, j$. Part (ii) is immediate from (i) and the definition.

From now on we suppose that $P$ is locally finite. Then we may define a linear map

$$
\partial: M \rightarrow M
$$

as follows: if $x \in P$ we put $\partial(x):=\sum y$ where the sum runs over all $y<x$ with $r(y)=r(x)-1$. Automorphisms of $P$ act linearly on $M$ and first we note that $\partial$ is an Aut $(P)$-map. Secondly, the map restricts to $\partial: M_{k} \rightarrow M_{k-1}$ for all $k>0$ and also to $\partial: M^{\mathcal{S}} \rightarrow M^{\mathcal{S}}$ for any $\mathcal{S} \subseteq P$. In particular, if $x \in P$ we may look at the restriction $\partial: M_{k}^{\{x\}} \rightarrow M_{k-1}^{\{x\}}$. We define the surjectivity index of $x$ as the largest integer $s(x)$ such that $\partial: M_{k}^{\{x\}} \rightarrow M_{k-1}^{\{x\}}$ is surjective for all $k \leq s(x)$.

Theorem 2.2 Let $(P, \leq)$ be a ranked locally finite poset, let $\mathcal{S} \subseteq P$ and let $G \subseteq$ Aut $(P)$. Then $n_{k-1}(G, \mathcal{S}) \leq n_{k}(G, \mathcal{S})$ for all integers $k$ with $k \leq \min \{s(x)$ : $x$ is a maximal element of $\mathcal{S}\}$.

Proof: As $\partial\left(A_{k}^{G}\right) \subseteq A_{k-1}^{G}$ we see that $\partial$ induces a map $\bar{\partial}: M_{k} / A_{k}^{G} \rightarrow M_{k-1} / A_{k-1}^{G}$. Let $B_{k}:=\left\langle x+A_{k}^{G}: x \in P_{k}^{\mathcal{S}}\right\rangle \subseteq M_{k} / A_{k}^{G}$ and define $B_{k-1}$ in the same way. From the definition of $P^{\mathcal{S}}$ we see that then $\bar{\partial}$ restricts to $B_{k} \rightarrow B_{k-1}$. Let $k \leq$ $\min \{s(x): x$ is a maximal element of $\mathcal{S}\}$ and let $z+A_{k-1}^{G} \in B_{k-1}$ with $z \in$ $P_{k-1}^{\mathcal{S}}$. By definition there then exists $h \in F P_{k}^{\mathcal{S}}$ with $\partial(h)=z$. Thus $\bar{\partial}\left(h+A_{k}^{G}\right)=$ $z+A_{k-1}^{G}$ and so $\bar{\partial}: B_{k} \rightarrow B_{k-1}$ is surjective. The remainder follows from Lemma 2.2 .

A related result appeared before in [14]. In many cases the surjectivity index is known or easily derived from known facts. If $P$ is the Boolean poset of all finite subsets of a set then the rank of $x \in P$ is $r(x)=\|x\|$ and the surjectivity index is the least integer above $r(x) / 2$, that is $s(x)=\lceil r(x) / 2\rceil$. This situation is very well understood, see for instance de Caen's elegant note [7].

Also in other product posets such as $3^{\Omega}$, more generally $k^{\Omega}$ and suitable finitary versions of such posets for infinite $\Omega$, we have $s(x)=\lceil r(x) / 2\rceil$. Indeed, $s(x)=$ 
$\lceil r(x) / 2\rceil$ occurs commonly for the surjectivity index. This applies to projective spaces, formed by the finite dimensional subspaces of a vector space over a finite field, were $r(x)$ is the affine dimension $\operatorname{dim} x$ of the subspace and $s(x)=\lceil\operatorname{dim} x / 2\rceil$. The same holds true in Desarguesian geometric lattices (i.e. direct products of projective spaces) and in unitary Peck posets, see Stanley [16, 18].

In the Boolean algebra, and many of the other examples above, a more subtle property can be used. We say that the element $x \in P$ is of binomial type if $\partial^{t-s}: M_{t}^{\{x\}} \rightarrow M_{s}^{\{x\}}$ is surjective for all $s \leq t$ with $s+t \leq r(x)$. (Note, there is no connection to the binomial posets considered elsewhere in the literature.) Now an obvious slight modification of the proof of Theorem 2.2 gives

Theorem 2.3 Let $(P, \leq)$ be a ranked locally finite poset, let $\mathcal{S} \subseteq P$ and let $G \subseteq \operatorname{Aut}(P)$. If all maximal elements in $\mathcal{S}$ are of binomial type then $n_{s}(G, \mathcal{S}) \leq$ $n_{t}(G, \mathcal{S})$ for any $s \leq t$ with $s+t \leq \min \{r(x): x$ is a maximal element of $\mathcal{S}\}$.

We now come to the proofs of the results in Section 1. As elements of the Boolean algebra are of binomial type Theorem 2.3 proves the Main Theorem in Section 1.

Proof of Theorem 1.4: Let $V$ be a vertex set and put $\Omega:=V^{\{2\}}$. Then $E \subseteq \Omega$ can be viewed as the graph with vertex set $V$ and edge set $E$, and if $E \supseteq E^{*}$ then the second graph may be thought of being obtained by deleting edges from $E$. In this way we may think of $\left(2^{\Omega}, \subseteq\right)$ as the graphs on $V$ ordered by edge deletion. Let $G=\operatorname{Sym}(V)$. Then $G$ acts naturally on $\Omega$ and $2^{\Omega}$. Clearly, two graphs $(V, E)$ and $\left(V, E^{*}\right)$ are isomorphic iff $E$ and $E^{*}$ belong to the same $G$-orbit. The result now follows from Theorem 2.3 or from the Main Theorem.

2.2 Amalgamation Classes: The two remaining corollaries, ostensibly about finite objects, are in fact proved via suitable infinite group actions. For this we consider classes of finite or countably infinite combinatorial structures which can be described as domains with relations on them. (A typical example is the class of graphs, considered as vertex sets with a single relation for adjacency.) Here we only give the briefest of account, for a fuller exposition see Section 5.6 in [8].

We need the concept of a relational language consisting of a collection of relation symbols each taking a finite number of arguments. A relational structure then is a domain (or set) together with relations defined on it and corresponding to the relational language. A class $\mathcal{C}$ of such structures is an amalgamation class if the following holds:

- Each $A$ in $\mathcal{C}$ is finite, $\mathcal{C}$ has at most countably many members up to isomorphism and $\mathcal{C}$ is closed under isomorphism and taking substructures, and 
- The amalgamation property holds: Whenever $A, B_{1}, B_{2}$ belong to $\mathcal{C}$ and there are embeddings $f_{i}: A \rightarrow B_{i}$ then there is some $C$ in $\mathcal{C}$ and embeddings $g_{i}: B_{i} \rightarrow C$ such that $g_{1} f_{1}=g_{2} f_{2}$.

Fraïssé's Theorem states that for each such amalgamation class there is a unique countable relational structure $\mathcal{R}$, called the Fraïssé limit, whose finite substructures are the members of $\mathcal{C}$. Furthermore, $\mathcal{R}$ is homogeneous in the sense that every isomorphism between members of $\mathcal{C}$ is induced by an automorphism of $\mathcal{R}$.

Proof of Theorem 1.3: Finite graphs form an amalgamation class whose Fraïssé limit is the random graph $\mathcal{R}$ on a countable set $\Omega$. Now apply the Main Theorem to $G:=\operatorname{Aut}(\mathcal{R})$ in its action on $\Omega$.

Proof of Theorem 1.2: Finite words in the alphabet $A$ also form an amalgamation class. We may think of its Fraïssé limit as the usual order $\leq$ on the rational numbers $\mathbb{Q}$ with a suitable random assignment $\alpha: \mathbb{Q} \ni r \mapsto \alpha_{r} \in A$. Let $G=\operatorname{Aut}(\mathbb{Q}, \leq, \alpha)$. Then the distinct words $x=\left(x_{1}, \ldots, x_{n}\right)$ with $x_{i} \in A$ correspond in one-to-one fashion to the (distinct!) $G$-orbits on the $n$-element subsets of $\mathbb{Q}$. Now apply the Main Theorem to $G$ in its action on $\mathbb{Q}$.

Other amalgamation classes of interest are bipartite graphs, $K_{n}$-free graphs or indeed finite posets, etc., see also Cameron's paper [9]. In each case one may apply the Main Theorem to obtain an orbit theorem for this amalgamation class.

\section{References}

[1] Biggs, N.L., Algebraic Graph Theory, Cambridge Univ. Press, London-NewYork, 1974.

[2] Billera, L.J. and Björner, A., Face numbers of polytopes and complexes, "Handbook of Discrete and Computational Geometry", (J.E. Goodman and J. O'Rourke, eds.), CRC Press, Boca Raton, New York, 1997, 291-310.

[3] Björner, A., The unimodal conjecture for convex polytopes, Bull. Amer. Math. Soc., 4 (1981), 187-188.

[4] Björner, A., Partial unimodality for f-vectors of simplicial polytopes and spheres, Contemporary Mathematics, 178, 45-54. Americ. Math. Soc. Providence, 1994.

[5] Björner, A., Garsia, A. and Stanley, R.P., An introduction to Cohen-Macaulay partially ordered sets, in Ordered Sets, Reidel, Dordrecht/Boston, 1982, pp. 583615. 
[6] Björner, A. and Ziegler, G.M., Broken circuit complexes: Factorizations and generalizations, J. Comb. Theory, 51 (1991), 96-126.

[7] de Caen, D., A note on the ranks of set-inclusion matrices, The Electronic Journal of Combinatorics 8 (2001), No 5.

[8] Cameron, P.J., Permutation Groups, LMS Student Texts 45, Cambridge University Press 1999.

[9] Cameron, P.J., Sequences realized by oligomorphic permutation groups, J of Integer Sequences, 3 (2000) Article 00.15.1.

[10] Handbook of Combinatorics, Graham, R., Grötschel, M. and Lovász, L. (editors) Elsevier 1995.

[11] Heron, A., Matroid polynomials, Combinatorics (Proceedings Conference on Combinatorial Mathematics), Southend-on-Sea, 1972, 164-202.

[12] Hibi, T., What can be said about pure O-sequences?, J. Comb. Theory, 50 (1989), 319-322.

[13] Livingstone, D. and Wagner, A., Transitivity of finite permutation groups on unordered sets, Math. Z., 90 (1965), 393-403.

[14] Mnukhin, V.B., Group actions on posets and a theorem of Livingstone and Wagner, Proceedings of the International Algebraic Conference in Memory of A.I. Shiershov, Novosibirsk, 1991. [In Russian].

[15] Read, R.C., An introduction to chromatic polynomials, J.Comb. Theory, 4 (1968), 52-71.

[16] Stanley, R.P., Some aspects of group actions on finite posets, J.Comb.Theory, 32A (1991), 241-277.

[17] Stanley, R.P., Log-concave and unimodal sequences in algebra, combinatorics and geometry, Annals of New York Acad. Sc. 576 (1989), 500-533.

[18] Stanley, R.P., Some applications of algebra to combinatorics, Discr. Appl. Mathematics 34 (1991), 132-161.

[19] Stanley, R.P., Combinatorics and Commutative Algebra, (Progress in Mathematics, 41), Birkhauser, 1983. 